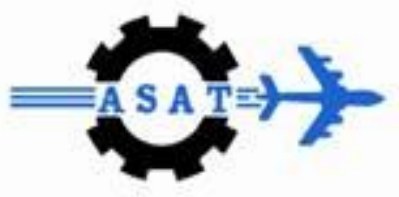

\title{
A Proposed Control System for an Autonomous Unmanned Ground Vehicle
}

\author{
A. Eliewa ${ }^{*}$, M. Atef*, M. Elhadad ${ }^{* *}$ and R. Khodary ${ }^{*}$
}

\begin{abstract}
This paper deals with a proposal of building up a control module to convert the APC M113 into a remotely controlled vehicle. The driving of the vehicle has been simplified and performed through a simple control lever similar to the computer joystick that is able to control the vehicle driving systems; steering and braking system, accelerator pedal, and gear shifting lever. The necessary sensors and actuators have been added to the conventional APC. These sensors and actuators nave been calculated and selected according the design diagram of the vehicle control system.
\end{abstract}

A microcontroller is used as a control unit, the required interfacing circuits are designed and necessary sensors and actuators are selected. LABVIEW Software ${ }^{\circledR}$ was used as the interface between the microcontroller and the real world.

Keywords: Unmanned systems, Electronic control, Microcontroller.

\section{Nomenclature}

$\begin{array}{ll}\mathrm{B} & \text { Track gauge. }(\mathrm{m}) \\ \mathrm{g} & \text { Gravitational acceleration, }\left(\mathrm{m} / \mathrm{s}^{2}\right) \\ \mathrm{h}_{\mathrm{c}} & \text { Height from the terrain to the bottom of the vehicle, }(\mathrm{m}) \\ \mathrm{n} & \text { Engine speed, (r.p.m) } \\ \mathrm{R} & \text { Radius of turning } \\ \mathrm{R}_{\mathrm{r}} & \text { Required radius of turning } \\ \mathrm{V} & \text { Actual vehicle speed } \\ \mathrm{V}_{\mathrm{r}} & \text { Required speed } \\ \mathrm{V}_{\mathrm{ot}} & \text { Overturning speed } \\ \mathrm{V}_{\mathrm{sk}} & \text { Side skid speed } \\ \mu & \text { Coefficient of lateral resistance to turning } \\ \omega & \text { Angular velocity of turning }\end{array}$

\section{Introduction}

In recent years, there has been a great world-wide interest in the development of autonomous ground vehicle system (AGVS) technologies due to their immediate potential in civil and military applications. [1]

\footnotetext{
* Egyptian Armed Forces

*** Higher Institute of Technology- $10^{\text {th }}$ of Ramadan
} 
The vehicle control system is a major component of the AGVS. It is a closed loop control system and consists of a controller, sensors as a feedback units, and actuators. The control system receives speed and radius of turning from a joystick, and generates appropriate commands for the actuators to drive the vehicle. The objective of the control system is for the tracked vehicle to safely follow the commanded speed and turning radius.

There are several attempts that have been made to address various issues for the control of tracked vehicles [2] - [7].

The main objective of this work is to demonstrate the feasibility of modifying an APC M113 - A2 conventional driving system to be remotely controlled. The proposal of adaptation of the control module to the vehicle is carried out through studying of the operations carried out by the driver, and introducing an interfacing control module to simplify these operations. Where a control system is proposed to take the decisions that are conducted to operate different actuators that drive the control mechanical linkages; so the driving is automated and simplified. The system is controlled through a control lever (similar to the computer joystick) which is able to control the vehicle operation directly or through a control link (in case of remote control) from a control station. The idea of components and control module parts is intended to be with a modular construction, compatible with vehicle available energy source, and adaptable to driving control linkages.

This paper will be divided into 3 sections, the $1^{\text {st }}$ section is an introduction and a short preview of the M113 specifications and characteristics, the $2^{\text {nd }}$ section gives a preview of the adaptation of the control module to the vehicle, finally the $3^{\text {rd }}$ section is the design of the control system, selecting of sensors and actuators, and the design of the control module.

The M113 is an American armored personal carrier (APC); it is powered by a Diesel engine of $210 \mathrm{hp}$, an automatic gearbox with three forward speeds and one reverse, and a steering differential for the steering and braking of the vehicle. The steering of the vehicle is employed by decreasing the speed of one track and increasing the speed of the other (using brakes inside the steering differential unit). The brakes are actuated mechanically and manually by a driver via two hand - command levers. The ranges of gears are selected manually but the gears within the manually selected range are shifted automatically. The specifications of the vehicle are listed in Table 1. [8] - [13]

Table 1 M113 Technical Data

\begin{tabular}{l|l}
\hline \hline \multicolumn{1}{c|}{ Item } & \multicolumn{1}{c}{ Data } \\
\hline Weight & 12 tons \\
Length & $4.8 \mathrm{~m}$ \\
Width & $2.7 \mathrm{~m}$ \\
Height & $2.7 \mathrm{~m}$ \\
Engine & $6 \mathrm{~V} 53$, Two - stroke Diesel engine, 6 cylinders V - type, water cooled, \\
& $210 \mathrm{hp}$. \\
Transmission & TX100 - 1, automatic gearbox with torque converter and lookup clutch, \\
& 3 forward speeds and 1 reverse. \\
\hline \hline
\end{tabular}

For manual driving, the accelerator pedal and the left and right brake levers are operated. Pressing / releasing the accelerator pedal increases / decreases the engine power output and therefore the vehicle speed. Pulling the left / right brake levers steers the vehicle to left / right. Pulling both the left and right brake levers reduces the vehicle speed. 


\section{Adaptation of control module to the vehicle}

The driver of that vehicle makes many operations such as increasing or decreasing the speed, gear shifting, and steering and braking. The existing remote controlled vehicles are mainly designed for electrical vehicle that are easy to be controlled. But there is much work to do with conventional vehicles equipped with internal combustion engines (ICE) to change it as a remote controlled one. It needs a control system for interfacing a simple control lever with vehicle manual control linkages (accelerator pedal, steering and brake levers, and gear shifting lever) to be similar to the electric vehicles.

The aim of control module is to operate as an interface between the driver control lever (or the remote control receiver unit) and the vehicle conventional mechanical control linkages; to do the job with a simplified manner.

The control module must be able to carry out the entire driver's work (Fig. 1): starting the engine, shifting gears for vehicle motion, sensing vehicle speed, steering, and braking, reverse direction of motion, opening and closing the ramp. Its design configuration takes into consideration the engine and transmission characteristics. [14]

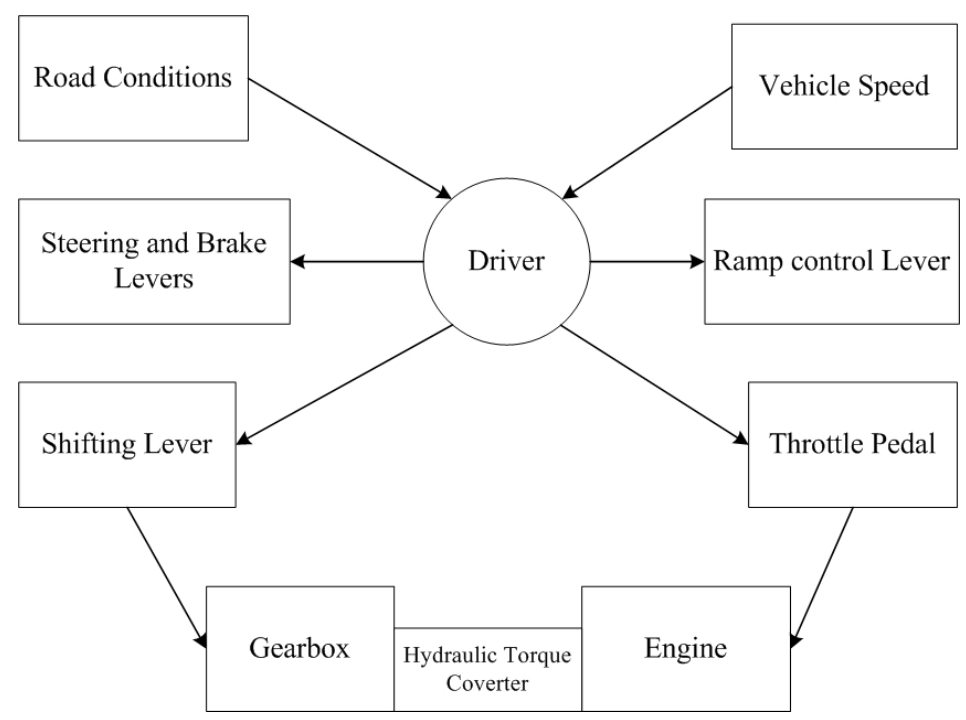

Fig. 1 Vehicle driver operations

\section{Proposed control system}

The proposed system algorithm takes the decisions and controls the actuators that drive the driving system according to the commands from the controller, the information based on vehicle characteristic data, and the initial conditions. Fig. 2 shows a simple block diagram of the proposed control system.

In this control system the driver force on the driving levers are replaced by a joystick and hydraulic actuators, these actuators need a complete hydraulic system to actuate. A controller is designed to deliver the prescribed actuating signal to the actuators according to the input signal. The necessary sensory elements are added to obtain the necessary correction. 


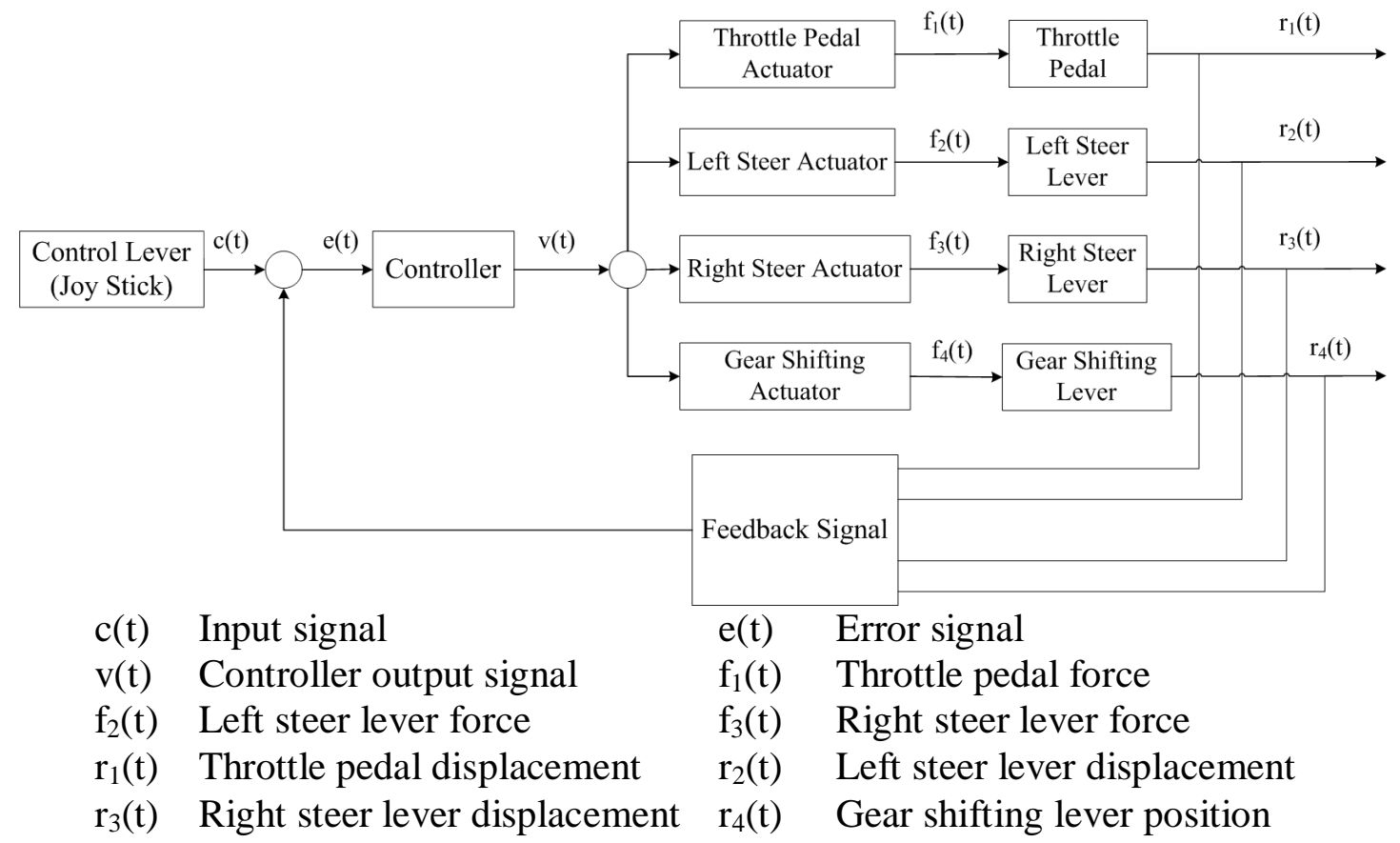

Fig. 2 Vehicle control system block diagram

\subsection{System main parts}

The proposed control system consists of steering and braking levers control system, gear shifting lever control system, throttle pedal control system, system sensors, system controller and interfacing circuits. The hydraulic control system consists of a pump that is the source of pressure energy, hydraulic cylinders to drive the levers, a directional control valves, check valves, pressure relief valve, to control the direction, flow rate, and the pressure respectively.

The controller receives the signal directly from the joystick or from the receiver (in case of remote operation), and according to the operating conditions of the vehicle; it takes a decision for operating the driving levers. The decision is transmitted to the hydraulic cylinders that connected to the levers.

To permit the manual operation of the vehicle, gate valves (7) are added to permit the driver to drive the vehicle manually; these gate valves are normally open for the autonomous driving, when closed the oil return back to the tank, so the driver can use the levers easily.

\subsubsection{System sensors}

The input signals are the needed radius of turning, the needed vehicle speed, and consequently the required gear range given by the driver (from the joystick), and the feedback signals from the sensors. For the steering and brake levers, the measured feedback signals is the speed of the right and left tracks of the vehicle that measured using digital optical encoders which measure the angular velocity of the right and left output shafts and then with some mathematical relations we can measure the radius of turning of the vehicle. The other feedback signal is the indication of the brakes that are completely applied or completely released using mechanical limit switches. For the gear shifting lever, the measured feedback signal is the gear range selector position that given using a six mechanical limit switches. For the accelerator pedal, the measured feedback signal is the engine crankshaft speed that given by a DC tachometer, and the pedal displacement using a linear variable differential transformer (LVDT). 


\subsubsection{Hydraulic subsystem}

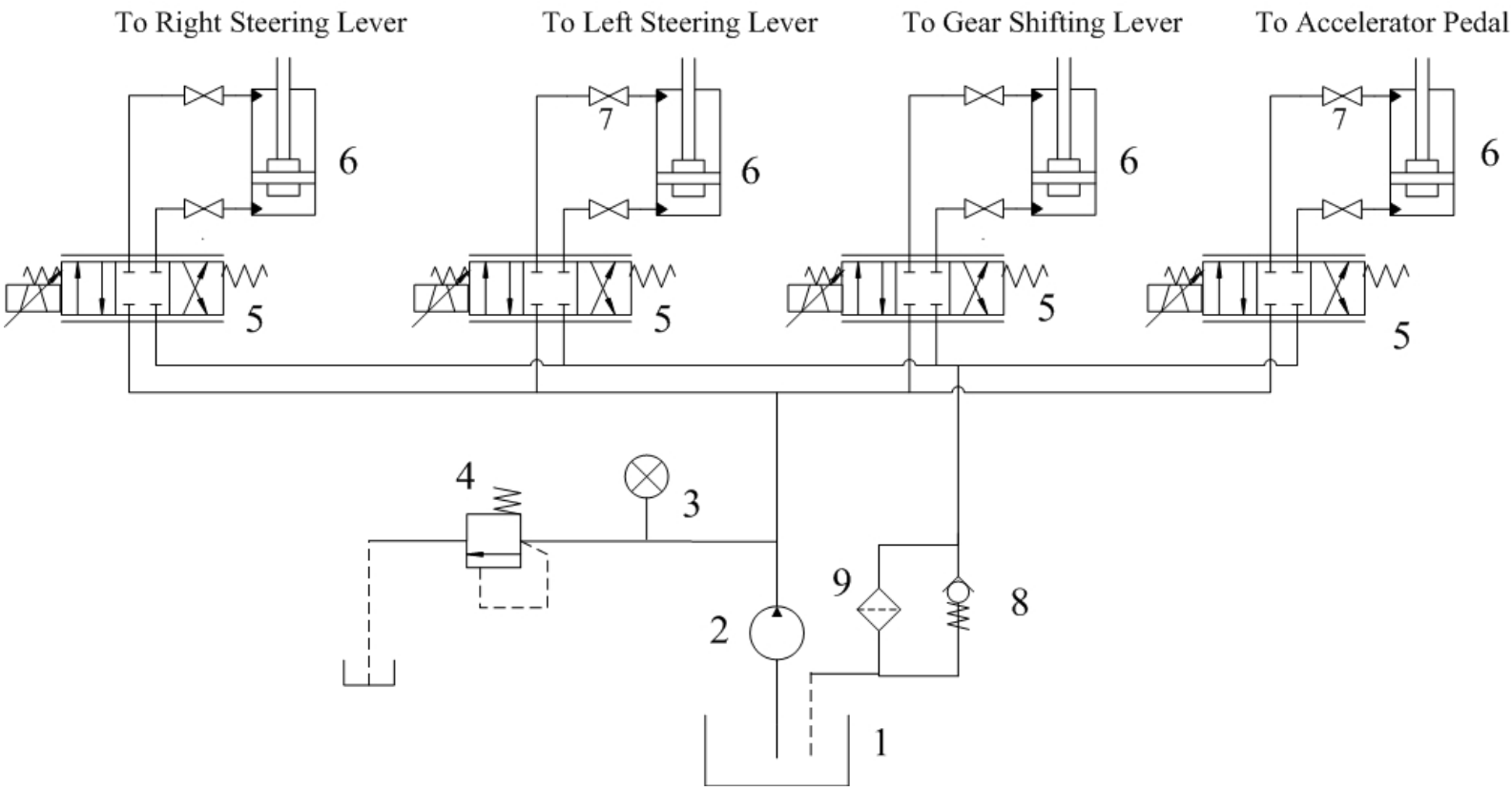

1 Fluid tank, 2 Pump, 3 Pressure indicator, 4 Relief valve, 5 Proportional directional control valves, 6 Hydraulic cylinders,

7 Gate valve, 8 Check valve, 9 Oil filter

\section{Fig. 3 Proposed hydraulic subsystem}

\subsubsection{System controller}

[14]The controller takes its input signals from the joystick, and its output signals are transmitted to the electro - hydraulic proportional directional control valves to actuate the hydraulic cylinders fixed to the levers. Sensors are detect the measured values required (engine speed, vehicle speed, position of gear shifting lever, etc), and send these measured values as a feedback signals to the controller. The used controller is a microcontroller 16F877A. The pin diagram of the microcontroller is shown in Fig. 4

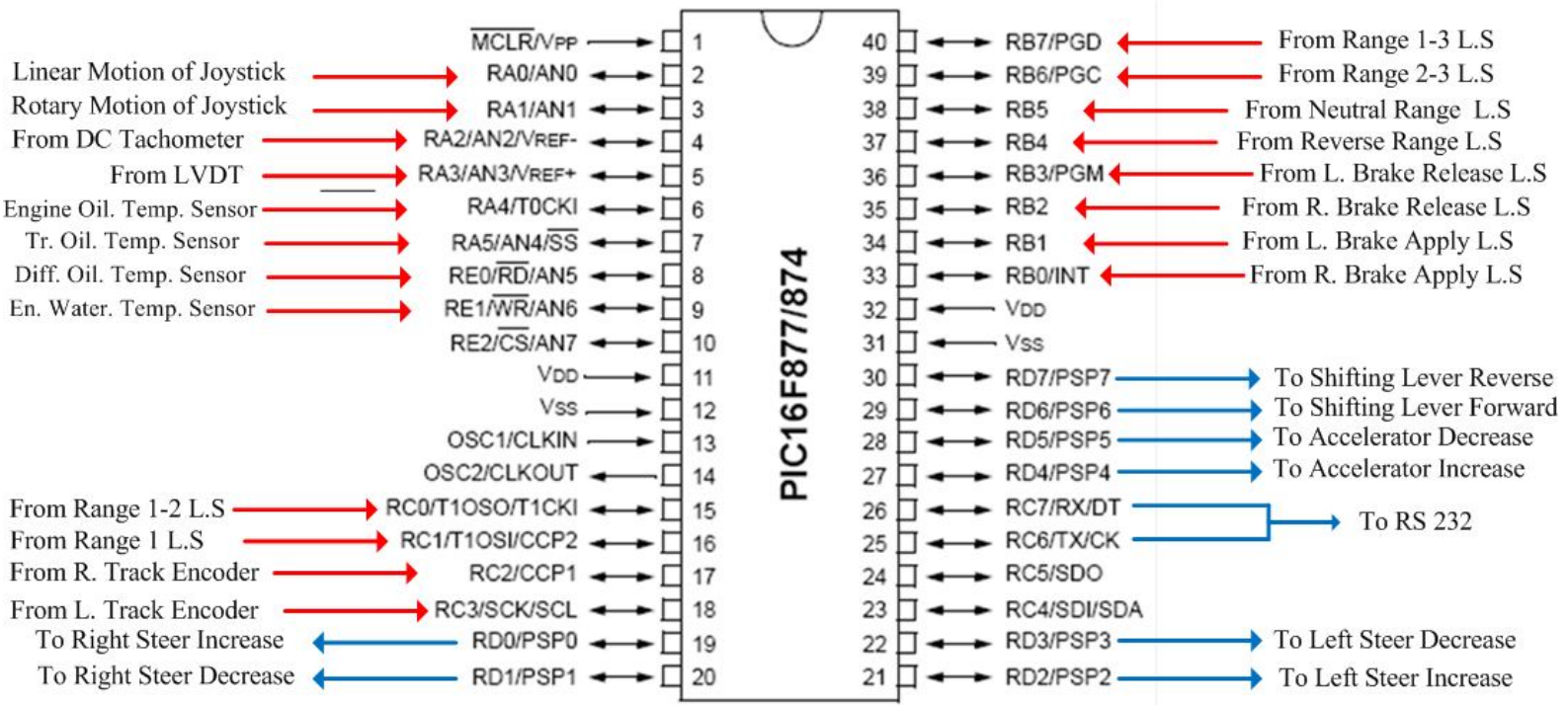

Fig. 4 Microcontroller pin diagram 
Autonomous driving is not only a driving by a remote control or a joystick, but also the driver instrument panel that located in the vehicle should exist as a panel for gauges and indicators. LABVIEW Software ${ }^{\circledR}$ has a great advantage of building up a front panel similar to the driver instrument panel. Building this panel will allow the driver of the vehicle using a joystick to follow the vehicle when it is remotely operated and can notes the vehicle speed, engine speed, engine oil, transmission oil, and differential oil temperatures.

\subsubsection{Description of control lever operation and positions}

The proposed control lever diagram shown in Fig. 5, the lever longitudinal movement changes the speed (increase, decrease or brake) and changes the direction of motion (forward (D) or reverse $(\mathbf{R})$ ) or stay in neutral $(\mathbf{N})$. Traversing the lever to right or left actuates the steering to right direction (RS) or left direction (LS). This mechanical movement is transformed into corresponding electrical signals to be interpreted to the control module. The joystick may exist in the control station and connected via wireless LAN to the microcontroller (in case of remote driving), or exists inside the vehicle and connected to the microcontroller via a cable.

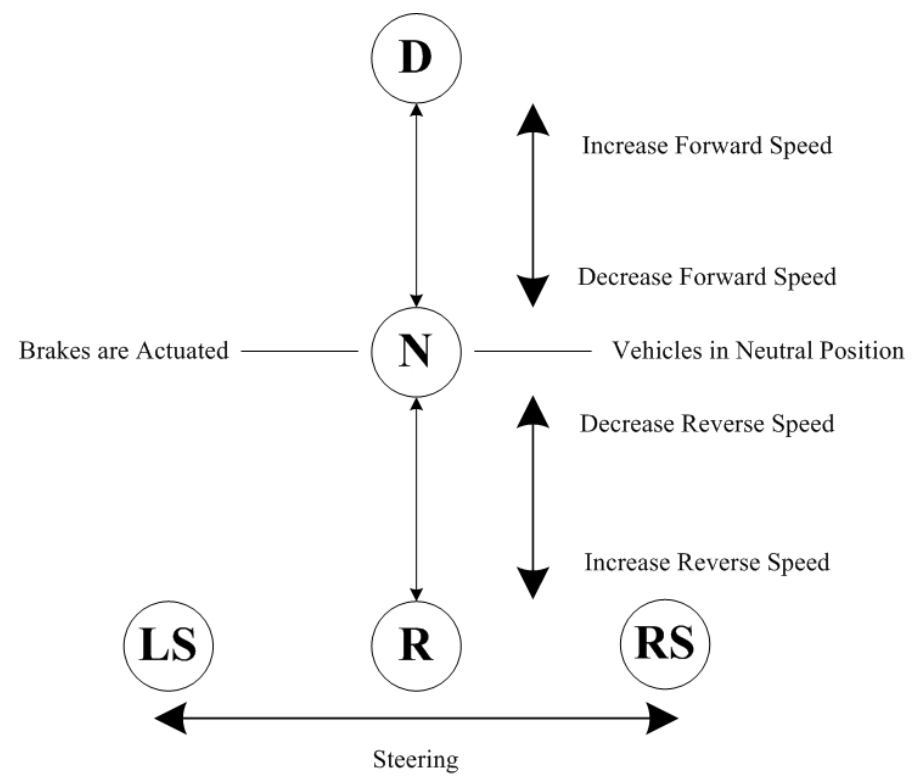

Fig. 5 Control lever operation diagram

\subsubsection{Configuration of control module}

The output electrical signals from the joystick are sent to other system control module, in which the required operations are activated: accelerator pedal operation, gear shifting, steering and braking as shown in Fig. 6 


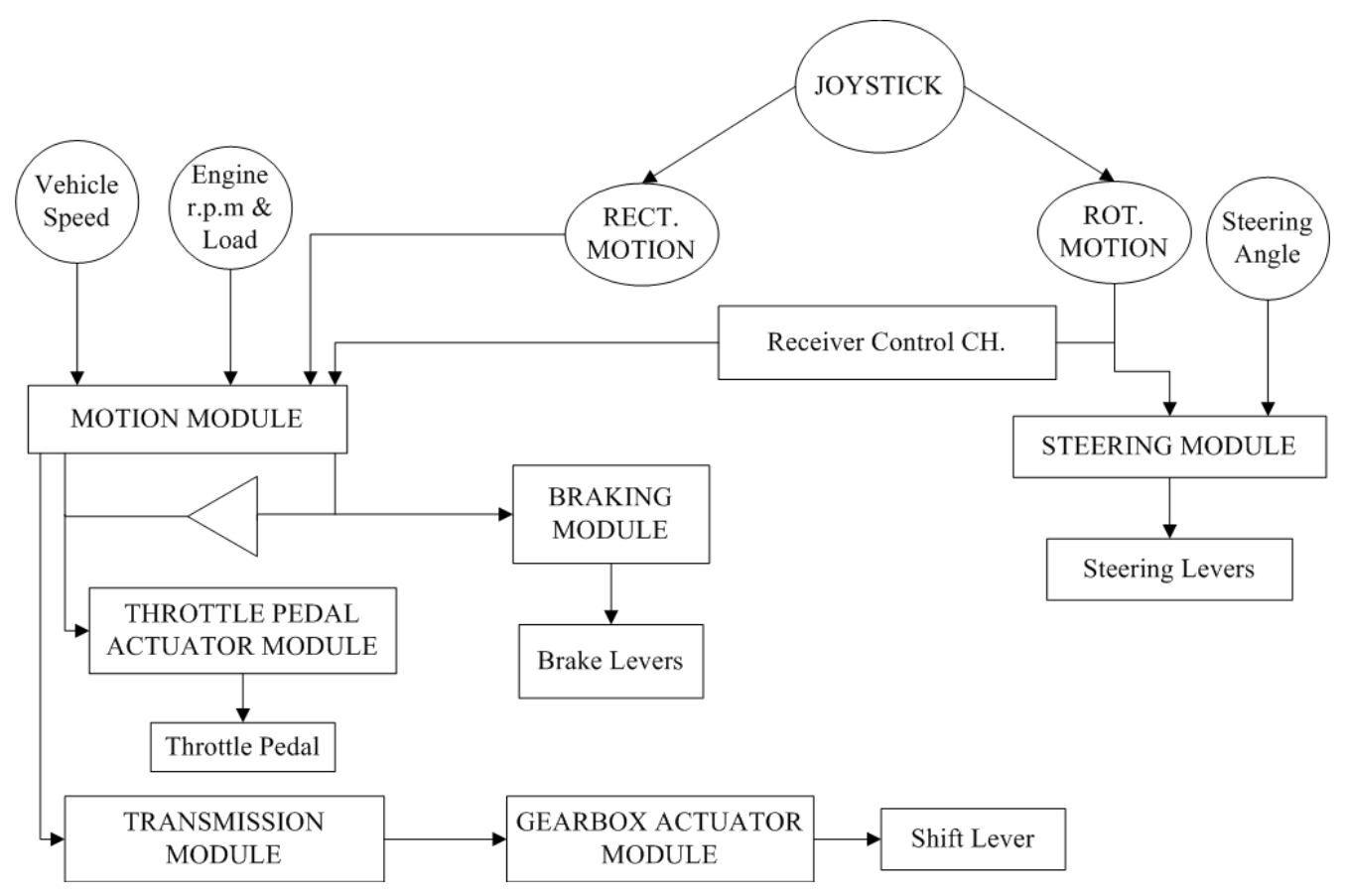

Fig. 6 Vehicle control module block diagram

\subsubsection{Operation of motion module}

The function of motion module is to take the decision of operating the vehicle mechanical levers according to the driving conditions and driver speed requirements.

The algoritm of operation of the mtion module is represented by the flowchart given in Fig. 7 . The inputs to the this module is the vehicle speed (V), the engine speed (n), the required speed given by the rectilinear motion of joystick $\left(\mathrm{V}_{\mathrm{r}}\right)$.

The outputs of this module is one of the following decisions:

i. Brake

i.e. apply the brake levers.

ii. Check for brake actuation

i.e. check if brake levers are actuated or not.

iii. Rel. Brakes

i.e. release the brake levers.

iv. Neutral

i.e. shift the shifting lever to the Neutral position.

v. Shift to $1-3$

vi. Shift to Reverse

i.e. shift the shifting lever to position $1-3$

vii. Increase speed

i.e. shift the shifting lever to the reverse position

viii. Decrease speed

i.e. increase the vehicle speed

i.e. decrease the vehicle speed

In the vehicles, it is impossible for the vehicle that moves in a specified direction (forward or reverse) to move in the other direction directly, the driver must stop the vehicle first and then shift to the required direction. The algorithm does that, if the vehicle moves in any direction and the driver moves the joystick lever in the opposite direction, the microcontroller stop the vehicle and then shift to the opposite direction. 


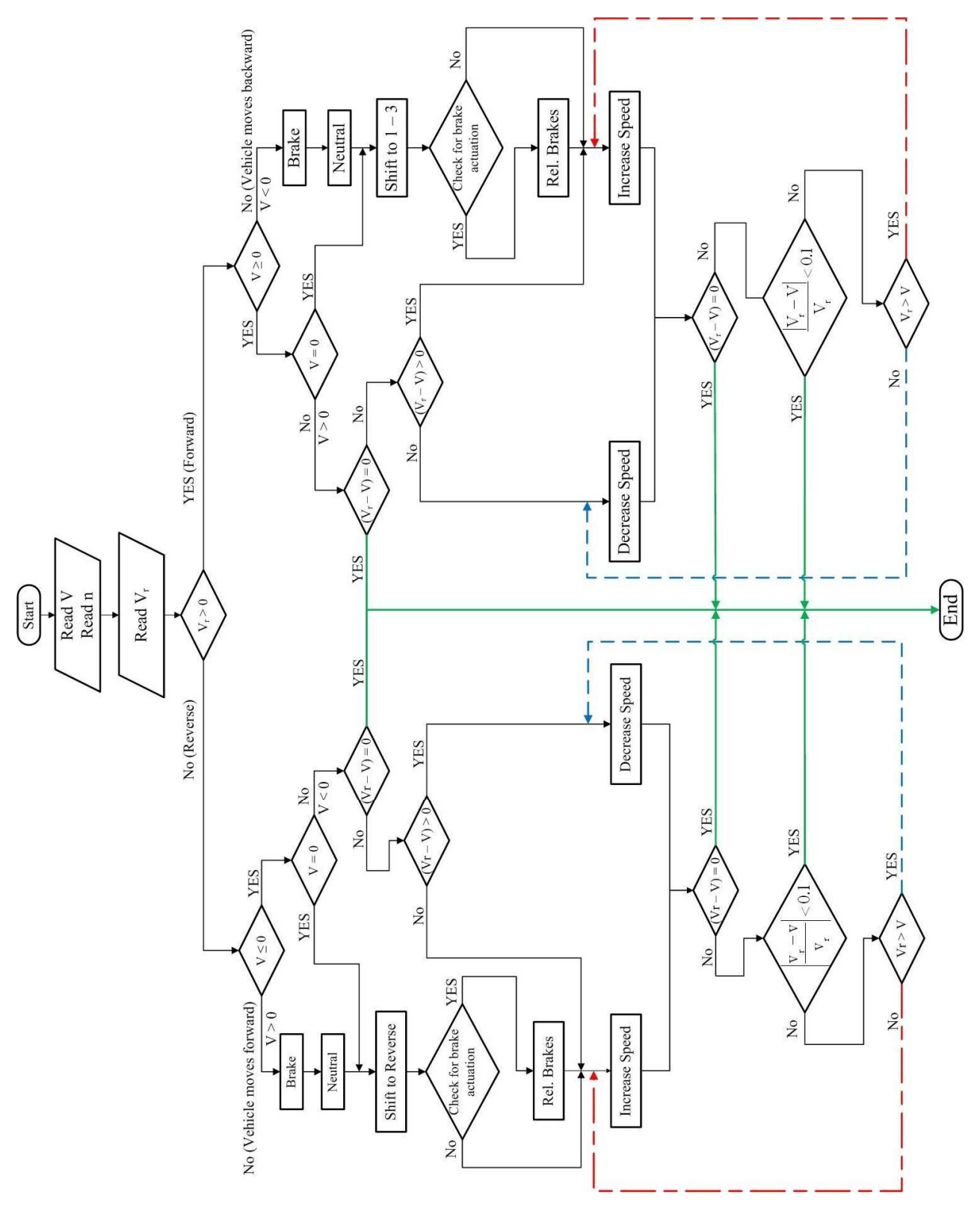

Fig. 7 Flowchart of motion module

\subsubsection{Operation of steering module}

The function of steering module is to take the decision of operating the vehicle mechanical levers according to the required radius of turning.

The algoritm of operation of the steering module is represented by the flowchart given in Fig. 8, the inputs to the this module is the speed of the inner track $\left(\mathrm{V}_{1}\right)$, the speed of the outer track $\left(V_{2}\right)$, and the required radius of turning given by the traversal motion of joystick $\left(\mathrm{R}_{\mathrm{r}}\right)$.

The outputs of this module is one of the following decisions:
i. Right Steer
i.e. make right steering (by moveing the right steer lever backward).
ii. Left Steer i.e. make left steering (by moveing the left steer lever backward).
iii. Increase Speed i.e. increase the vehicle speed
iv. Brake i.e. apply the brake levers 
In the steering of the tracked vehicles, there are two critical parameters, the overturning speed $\left(\mathrm{V}_{\mathrm{ot}}\right)$ and skid speed $\left(\mathrm{V}_{\mathrm{sk}}\right)$ that the vehicle will rollover or skid if it turns with these speeds at a specific radius of turning. For the vehicle neither rollover nor skid, the speed of turning should be greater than the skid speed and less than the overturning speed.

As shown in Fig. 8, the controller takes the required radius of turning from the joystick, then calculates $\mathrm{V}_{\text {ot }}$ and $\mathrm{V}_{\text {sk }}$ before operating the steering levers, if the overturning speed is greater than the vehicle speed $\left(\mathrm{V}_{\mathrm{o}}\right)$, the algorithm will brake the vehicle until $\mathrm{V}_{\mathrm{ot}}$ be less than $\mathrm{V}_{\mathrm{o}}$, and if the skid speed will be less than the vehicle speed, the algorithm will increase the speed until $\mathrm{V}_{\text {sk }}$ will be greater than the $\mathrm{V}_{\mathrm{o}}$.

The microcontroller calculates the turning parameters according to the following equations: [15]

The track velocities $\left(\mathrm{v}_{1}, \mathrm{v}_{2}\right)$ :

$$
v=\omega^{*} r_{d . s}
$$

where $\omega 1, \omega 2$ are measured using the optical rotary encoders fixed to the driving sprockets.

Then we can calculate the radius of turning of the carrier from the following relation:

$$
R=\frac{v_{2}}{v_{2}-v_{1}} B
$$

The angular velocity of turning $(\boldsymbol{\omega})$ :

$$
\omega=\frac{v_{2}}{3.6 R}
$$

The speed of turning $\left(\mathbf{v}_{\mathbf{o}}\right)$ :

$$
v_{o}=3.6 \omega\left(R-\frac{B}{2}\right)
$$

The critical overturning velocity $\left(\mathrm{v}_{\mathrm{ot}}\right)$ :

$$
v_{o t}=36 \sqrt{\frac{(R-B / 2) g \cdot B}{2 h_{c}}}
$$

The critical side skid velocity $\left(\mathrm{v}_{\mathrm{sk}}\right)$ :

$$
v_{s k}=3.6 \sqrt{\left(R-\frac{B}{2}\right) \mu g}
$$



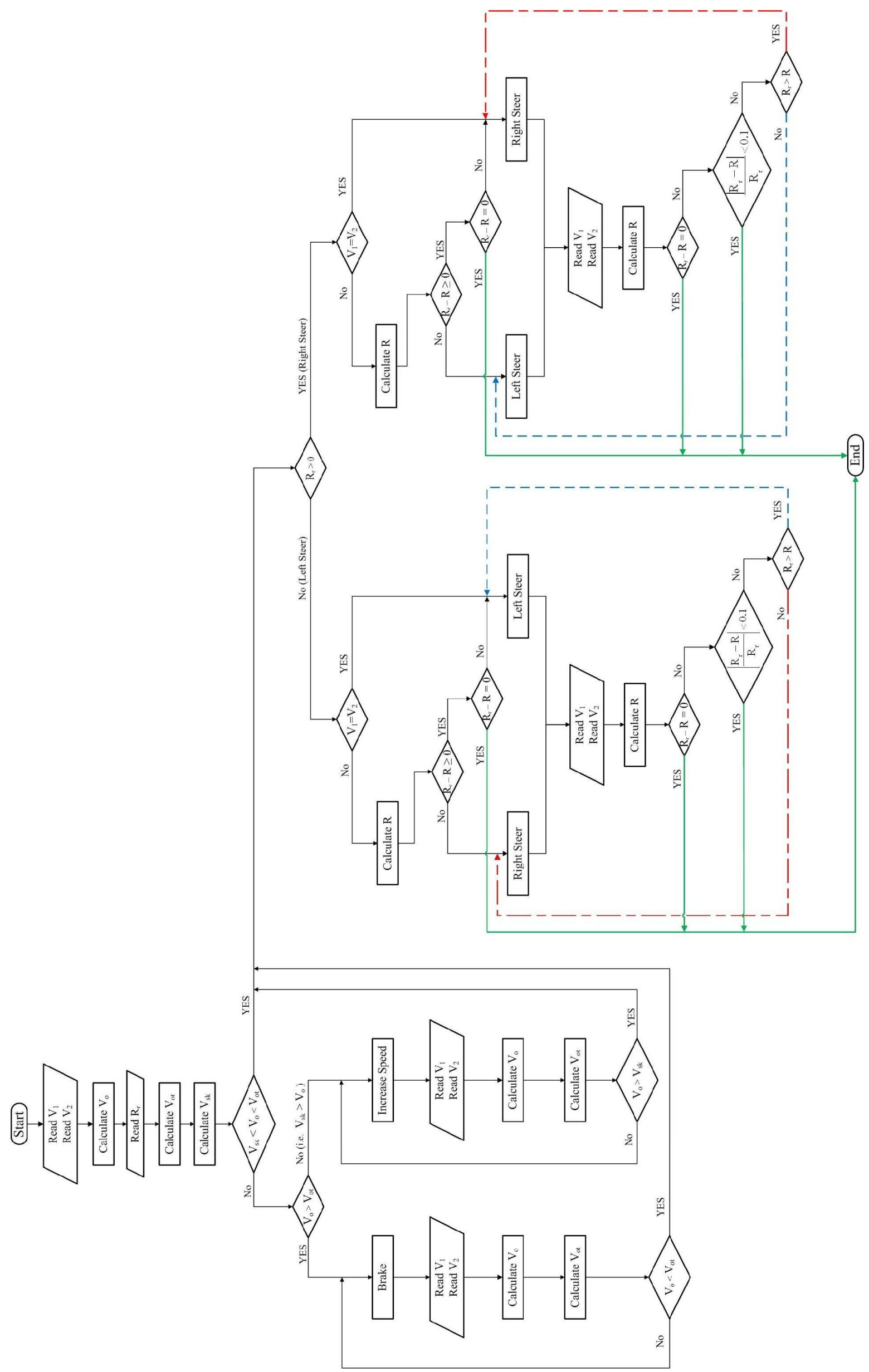

Fig. 8 Steering module flowchart 


\subsubsection{LABVIEW interface}

Serial communication is a popular means of transmitting data between a computer and a peripheral device such as a programmable instrument, or even another computer. Serial communication uses a transmitter to send data, one bit at a time, over a single communication line to a receiver. this method used when data transfer rates are low or data must transfer over long distances. Serial communication requires that you specify four parameters: the baud rate of the transmission, the number of data bits encoding a character, the sense of the optional parity bit, and the number of stop bits. Each transmitted character is packaged in a character frame that consists of a single start bit followed by the data bits, the optional parity bit, and the stop bit or bits. [16]

In this work, the baud rate used is 9600; the transferred data is 8 bit, with no parity and 1 stop bit. The designed front panel is shown in Fig. 9, while the block diagram is shown in Fig. 10
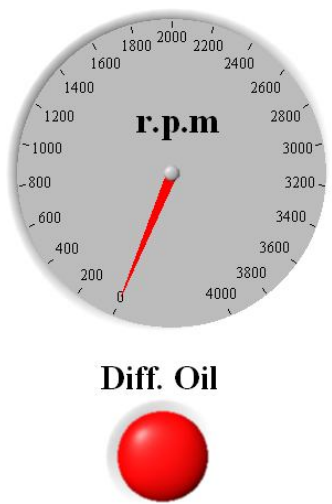

Hi - Temp.
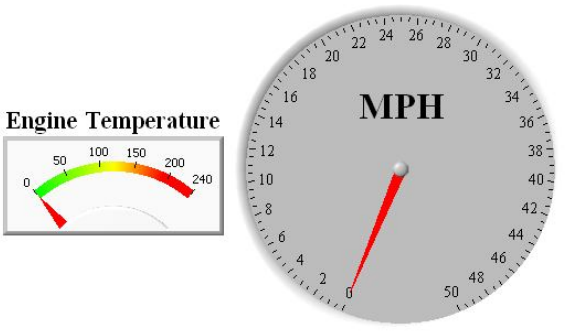

Engine Oil

Trans. Oil

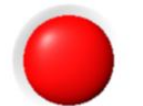

Hi - Temp.

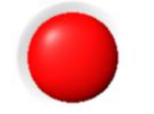

Hi - Temp. - Lo Press.

Fig. 9 Driver panel designed by LABVIEW

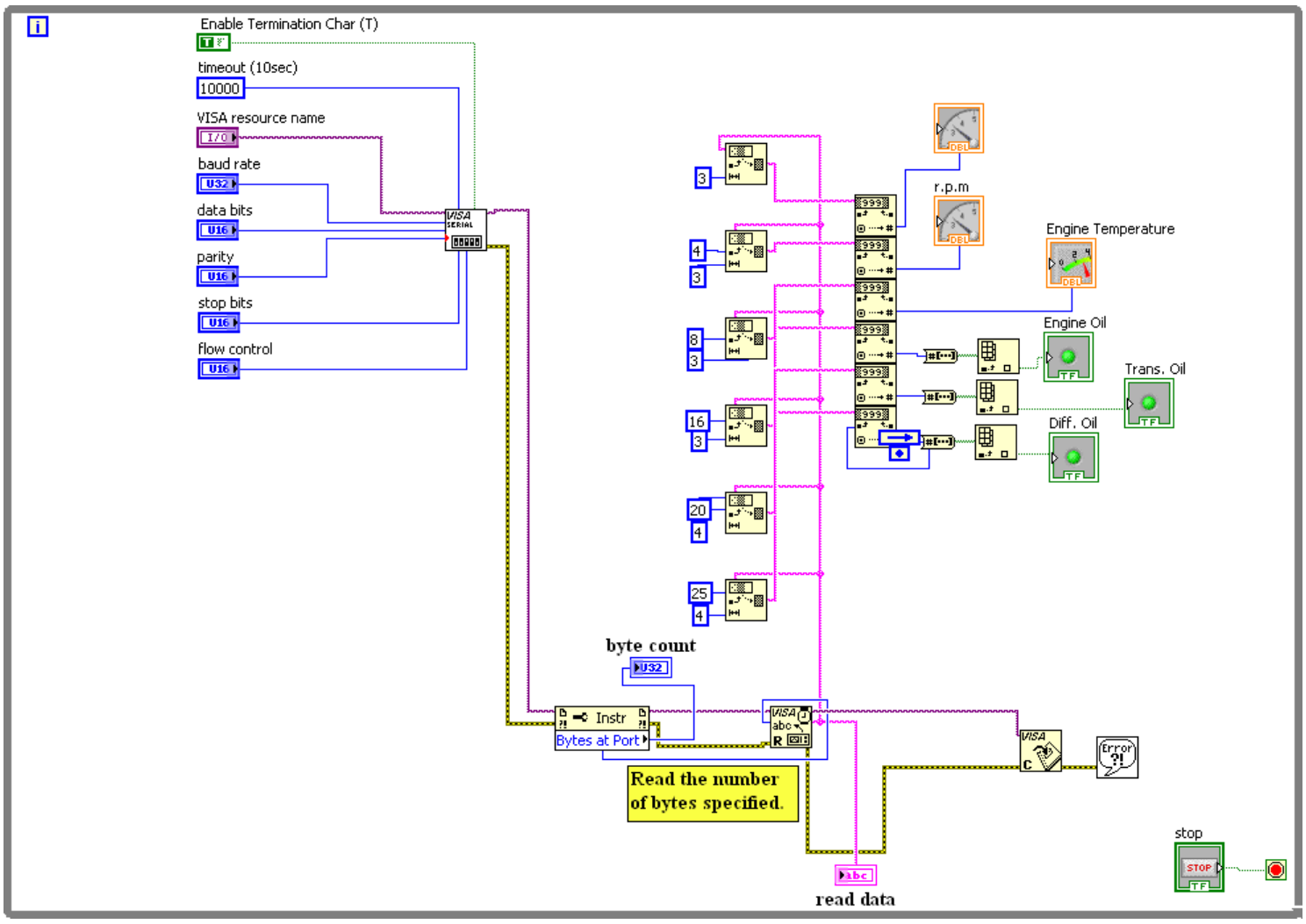

Fig. 10 LABVIEW block diagram 


\subsubsection{Interfacing circuits}

Some sensors and actuators need a required input voltage to operate, the vehicle existing electrical system depends on its batteries, they are 2 batteries connected in series, each has $12 \mathrm{~V}-100 \mathrm{Ah}$, so that some interfacing circuits are needed to supply these systems with the necessary voltage required to operate. These systems are the electro - hydraulic proportional directional control valves, the joystick, the 2 optical rotary encoders and the limit switches, and the warning lights of engine oil, transmission oil, and differential oil temperatures.

\subsubsection{Interfacing circuit for the electro - hydraulic P.D.C.V}

This valve supply voltage is a $24 \mathrm{~V}$ DC that is supplied from the vehicle's batteries. The control command signal is $0: \pm 10$ VDC. The command control signal is the signal that control the displacement of the spool, increasing the voltage will increase the speed of the cylinders' extending or retracting, the negative voltage for the motion of the spool in the other direction. In this work we will get the control signal to be \pm 5 VDC for operating the cylinders. The selected P.D.C.V is equipped with a built in controller that combines all necessary functions for the optimal operation of the valve. It takes the voltage supply signal and the command signal. The valve controller has a built in feedback to the spool displacement and position. The scheme of the P.D.C.V controller is shown in Fig. 11

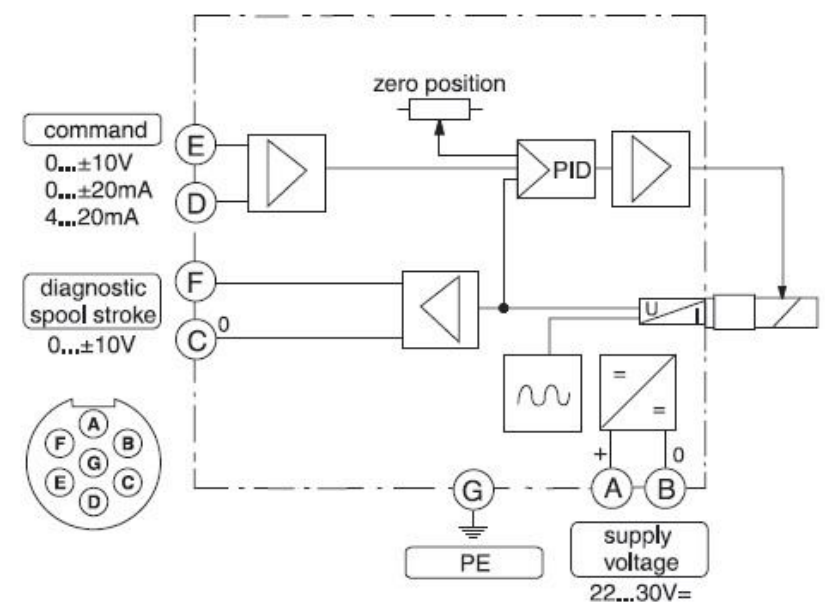

Fig. 11 The control circuit of the P.D.C.V

Pins A, B are connected to the vehicle's batteries; pins D, E are the input pins of the command signal, these pins are connected to the microcontroller, the microcontroller output signal is digital ( 0 or 1$)$ and the required command signal is analogue $(0$ or $\pm 5 \mathrm{~V})$. To solve this problem, a simple circuit should be designed as shown in Fig. 12.

Current flows from the battery to the regulators; regulator L7805 regulates the voltage to be $+5 \mathrm{VDC}$ and connected to one of the vehicle batteries, while regulator L7905 regulates the voltage to be -5 VDC and connected to the other battery. The output of regulator L7805 is fed to the normally open control relays (K1, K2, K3, K4), and the output of regulator L7905 is fed to the normally open control relays (K4, K5, K6, K7). These relays are energized by means of the output digital signal comes from the port $\mathrm{D}$ of microcontroller. When the output of one of the channels (RD0, RD2, RD4, RD6) is one, the inverter 7404N invert it to be zero, so the relay coil energized and current flows from regulator L7805 to the valve solenoid through the control relay. If the output of the channel is zero, the inverter inverts it to be one, the relay coil not energized and so there is no current flow to the solenoid and it will stop at this position. The same will do for the other relays but the current flows from the regulator L7905, and the valve solenoid will reverse its direction of motion causing a specified hydraulic cylinder retraction 


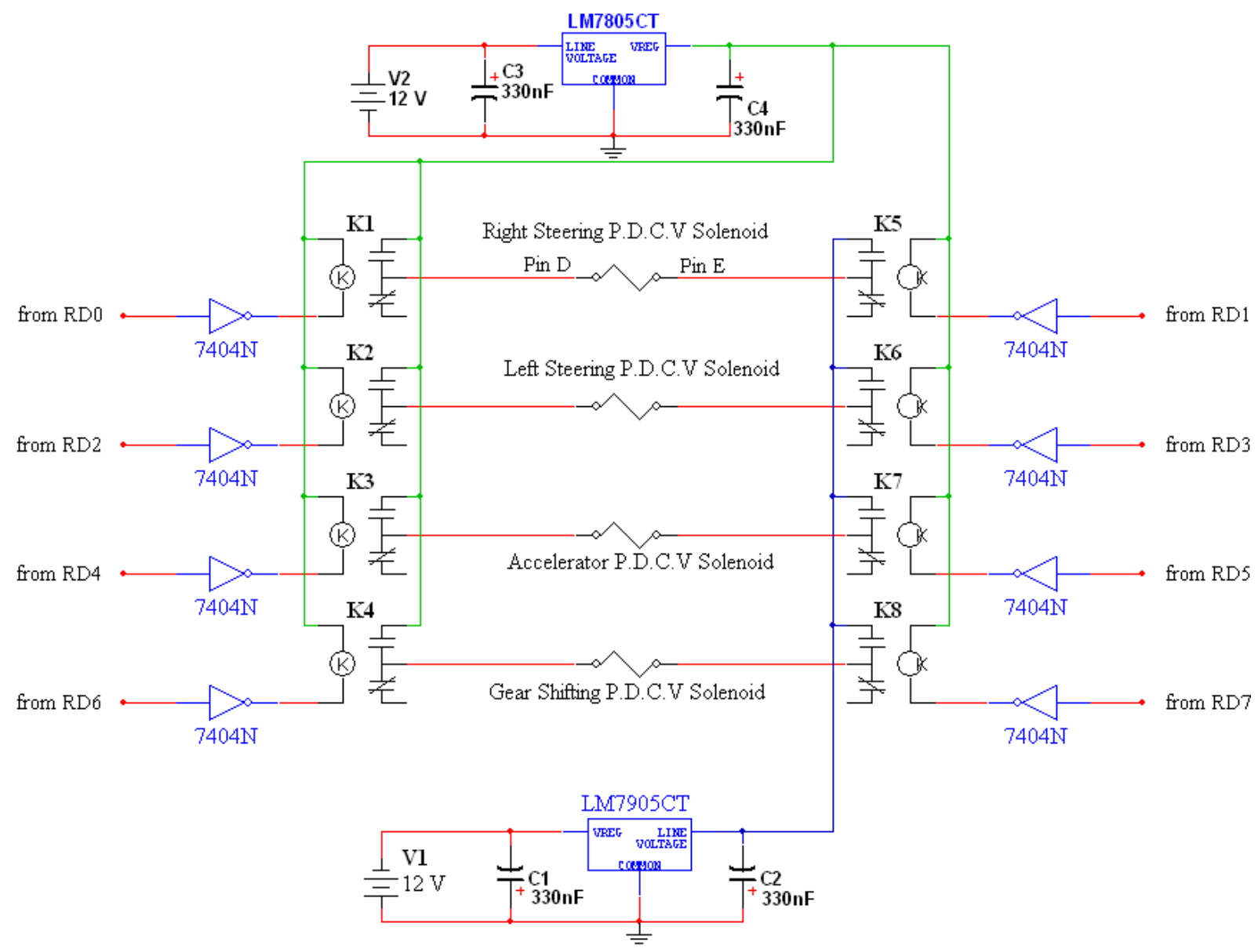

Fig. 12 P.D.C.V interfacing circuit

\subsubsection{Interfacing circuit for the joystick}

The joystick needs a 5VDC to operate, A +5VDC from the voltage regulator 7805 is fed to the (outside) terminals of the joystick pot as shown in Fig. 13

The joystick has two rotary potentiometers $\mathbf{R} \mathbf{1}$ for the joystick linear motion and $\mathbf{R} \mathbf{2}$ for joystick traversal motion. The outputs of these two potentiometers are fed as an analogue input to the microcontroller to channels (RA0 and RA1).

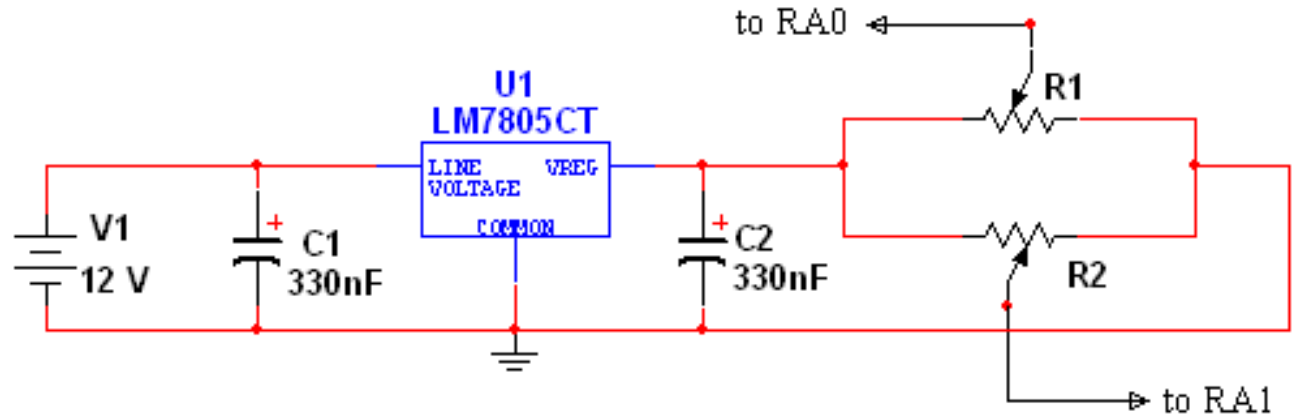

Fig. 13 Joystick interfacing circuit

\subsubsection{Interfacing circuit for the optical encoders}

Similarly, the optical rotary encoders need a 5VDC supply voltage, so an interfacing circuit similar to the circuit of the joystick is designed as shown in Fig. 14. The output of the encoders are fed to channels (RC2, RC3). 


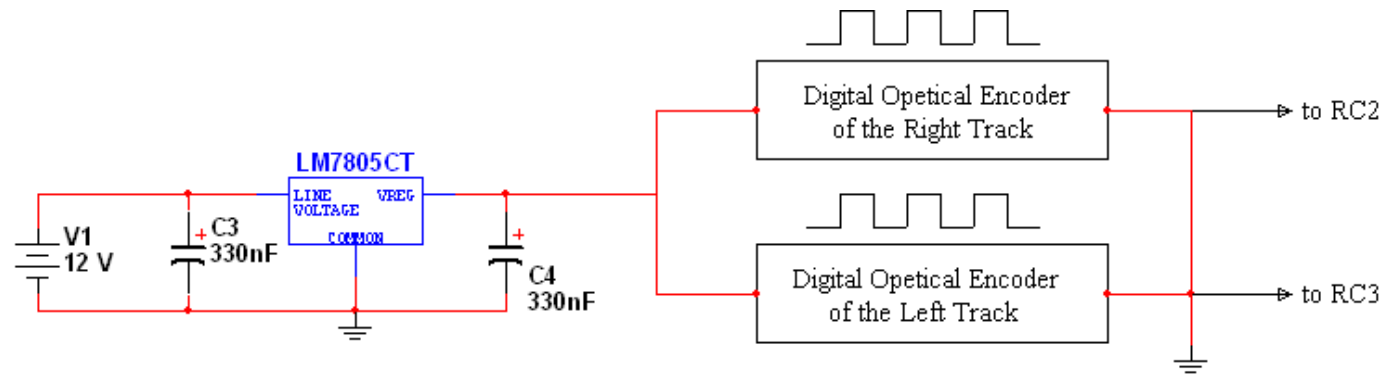

Fig. 14 Digital optical encoders interfacing circuit

\subsubsection{Interfacing circuit for the limit switches.}

Fig. 15 shows the interfacing circuit of the limit switches to the control circuit. In the proposed control system, there are ten limit switches. The regulated +5VDC voltage is applied to the micro switches' terminals through two $220 \Omega$ pull up resistors as shown. The sensors' signals are digital signals, which are fed to the digital input channels (RB0, RB1, RB2, RB3, RB4, RB5, RB6, RB7, RC0, and RC1) of the microcontroller.

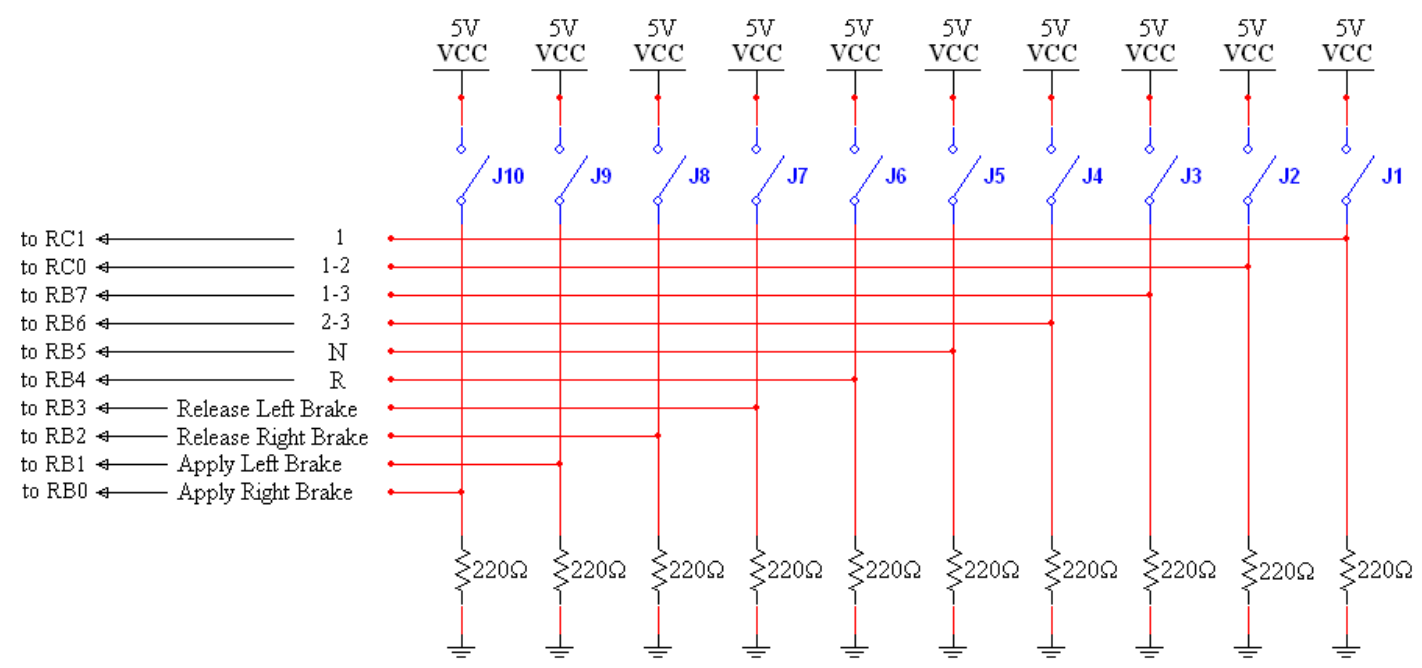

Fig. 15 Limit switches interfacing circuit

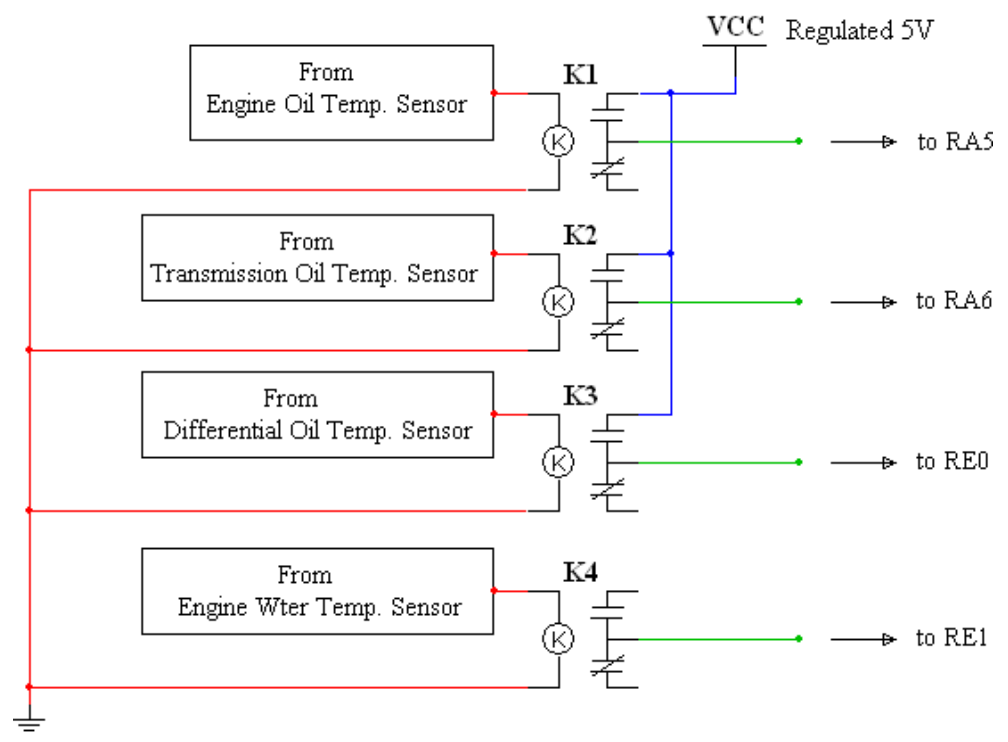

Fig. 16 Temperature sensors interfacing circuits 


\subsubsection{Interfacing circuit for the warning indicators}

The indicators used in the vehicle, supplied with 12 VDC from vehicle battery, so using three 12 VDC relays, when current flows from these temperature sensors to the relays, they energized and the regulated 5VDC will supply to the microcontroller as shown in Fig. 16.

\section{References}

[1] H. Durrant - Whyte, "A Critical Review of the State of the Art in Autonomous Land Vehicle Systems and Technology", SANDIA REPORT 2001, Sandia International Laboratories, USA, 2001

[2] N. K. Boon, T. H. Choo, and C. C. Wah, "Autonomous Unmanned Ground Vehicle and Indirect Driving",

[3] Z. Gong, J. I. Guzman, S. J. Scheding, D. C. Rye, G. Dissanayake, H. D. Whyte "A Heuristic Rule - Based Switching and Adaptive PID Controller for a Large Autonomous Tracked Vehicle: Form Development to Implementation", Proceedings of the 2004 IEEE, International Conference on Control Applications, PP 1277 - 1277, Taiwan. April 2004.

[4] I. Schwartz, "PRIMUS Autonomous Driving Robot for Military Applications", Proceedings of SPIE, Vol. 4024, PP 313 - 325, Aero Sense 2000, Unmanned Ground Vehicle Technology II, April 2000.

[5] J. G. Morillon, and O. Lecointe, "SYRANO: A Ground Robotic System for Target Acquisition and Neutralization", Proceedings of SPIE, Vol. 5083, Aero Sense 2003, Unmanned Ground Vehicle Technology V, April 2003.

[6] G. G. Wang, S. H. Wang and C. W. Chen "Design of a Turning Control for a Tracked Vehicle", IEEE Control System mMagazine, Vol. 10, No. 3 P.P 122 - 125, April 1990.

[7] J. J. Nitao and A. M. Paradi, "A Teal - Time Reflexive Pilot for an Autonomous Land Vehicle”, IEEE Control System Magazine, Vol. 6, No. 1, PP 14 - 23, Feb. 1986.

[8] www.FAS.org.

[9] "Unit Maintenance Technical Manual of APC - M113 - A2" (TM 9 - 2350 - 261 - 20 1,) Washington, Headquarters of US Army, July 1997.

[10] “Operator's Manual of APC - M113-A2”, (TM 9 - 2350 - 261 - 10), Washington, Headquarters of US Army, July 1997.

[11] "Technical Manual for Direct Support and General Support Maintenance - engine of APC M113 - A2", (TM 9 - 2815 - 205 - 34), Washington, Headquarters of US Army, Sep. 1991

[12] "Technical Manual for Direct Support and general Support Maintenance - Automatic Transmission of APC M113-A2", (TM 9 - 2520 - 254 - 34), Washington, Headquarters of US Army, Sep. 1991

[13] "Technical Manual for Direct Support and general Support Maintenance - Steering Differential of APC M113-A2", (TM 9 - 2520 - 238 - 34), Washington, Headquarters of US Army, Sep. 1991

[14] M. A. Elhaddad, "Proposal of Remote Drive Ground Vehicles", Proceedings of 9th AMME Conference, Vol.2, PP 733 - 744, Cairo, May 2000.

[15] Theory of Tanks", printed Lecture, M.T.C

[16] www.ni.com. 